\title{
White Matter Microstructure in Adolescents and Young Adults With Non-Suicidal Self-Injury
}

\author{
Melinda Westlund Schreiner ${ }^{1 *}$,Bryon A. Mueller ${ }^{2}$, Bonnie Klimes-Dougan ${ }^{3}$, \\ Erin D. Begnel ${ }^{3}$, Mark Fiecas ${ }^{4}$, Dawson Hill ${ }^{2}$, Kelvin O. Lim ${ }^{2}$ and Kathryn R. Cullen ${ }^{2}$ \\ ${ }^{1}$ Department of Psychiatry, University of Utah, School of Medicine, Salt Lake City, UT, United States, ${ }^{2}$ Department of \\ Psychiatry and Behavioral Sciences, University of Minnesota Medical School, Minneapolis, MN, United States, ${ }^{3}$ Department \\ of Psychology, University of Minnesota College of Liberal Arts, Minneapolis, MN, United States, ${ }^{4}$ Division of Biostatistics, \\ University of Minnesota School of Public Health, Minneapolis, MN, United States
}

Background: Non-suicidal self-injury (NSSI) is a growing public health concern that commonly begins in adolescence, and can persist into young adulthood. A promising approach for advancing our understanding of NSSI in youth is to examine white matter microstructure using diffusion magnetic resonance imaging (dMRI).

Method: The present study examined whole-brain group differences in structural connectivity (as measured by generalized fractional anisotropy [GFA]) between 28 female adolescents and young adults ages 13-21 years with NSSI and 22 agematched healthy controls $(\mathrm{HC})$. We also explored the association between clinical characteristics including NSSI severity and duration, impulsivity, emotion regulation and personality traits within the NSSI group and GFA of the uncinate fasciculus and cingulum.

Results: Compared to the HC group, participants with NSSI had lower GFA in several white matter tracts, including the uncinate fasciculus, cingulum, bilateral superior and inferior longitudinal fasciculi, anterior thalamic radiation, callosal body, and corticospinal tract. When controlling for depressive symptoms, the NSSI group showed an association between NSSI duration (time since initiating NSSI behavior) and lower GFA in the left cingulum. Higher levels of attentional impulsivity were related to lower GFA in the left uncinate fasciculus within the NSSI group.

Conclusions: We found evidence suggesting widespread white matter microstructure deficits in adolescents and young adults with NSSI versus HC. We also report inverse associations between white matter integrity and clinical characteristics (duration of NSSI and attentional impulsivity). These white matter microstructural deficits may represent a possible neurobiologically-based vulnerability to developing maladaptive coping mechanisms, such as NSSI. Additionally, results suggest that this white matter disorganization may either worsen with prolonged engagement in NSSI or predict persistent NSSI; thereby highlighting the importance of early intervention targeting this behavior.

Keywords: non-suicidal self-injury, neuroimaging, fractional anisotropy, uncinate fasciculus, cingulum, adolescents 


\section{INTRODUCTION}

Non-suicidal self-injury (NSSI), or the purposeful act of harming oneself without suicidal intent, commonly begins in adolescence and is associated with negative outcomes such as persistent psychopathology and suicide (1-3). Research examining the neurobiological correlates of NSSI is necessary to guide the development of biologically-informed interventions. Given that it is a particularly sensitive developmental period of significant neurobiological changes and the onset of NSSI, research may benefit from focusing on adolescence in particular (4-6).

The efficient transmission of neural signals depends in part on the organization and integrity of white matter fiber bundles and the structural characteristics of the myelin sheath that surrounds the body of an axon. These characteristics help to facilitate and constrain neuronal communication, thereby enhancing efficiency of neural functioning (7). Diffusion MRI (dMRI) is a brain imaging method that measures the diffusion of water molecules within the white matter of the brain. The dMRI metric of fractional anisotropy (FA) has traditionally been used used to estimate white matter organization. FA produces a value between zero and one, in which zero reflects complete isotropy (diffusion is not at all restricted or is restricted equally in all directions) and one reflects anisotropy (diffusion is confined to a particular direction). The assumption is that FA values reflect characteristics of white matter microstructure, such as myelination and directionality or coherence of white matter fiber bundles [see (8) for review]. In this case, higher FA values are typically interpreted as reflecting more optimal organization and integrity of white matter.

One challenge in dMRI research has been that when an MRI voxel captures multiple crossing fibers, the FA measurement will be artifactually low (9). To address this issue, a recent advance in dMRI research has been to use High Angular Resolution Diffusion Imaging (HARDI) acquisition, which allows for analysis strategies that may resolve multiple fiber directions in a voxel $(10,11)$. This can be accomplished by using spherical harmonization to calculate the Orientation Distribution Function (ODF), which is then used to estimate Generalized Fractional Anisotropy [GFA; (12)]. Similar to FA, higher GFA values indicate greater directionality of diffusion. GFA improves on the standard tensor model by being less susceptible to the effects of crossing or "kissing" white matter fibers $(13,14)$.

Only one study to date has used dMRI to examine white matter in patients with a history of self-injury (15). This study found that women with borderline personality disorder (BPD) and a history of self-injury had lower FA within the inferior frontal lobe compared to controls (15). However, study limitations included a small sample size $(\mathrm{n}=9 \mathrm{BPD}$ and 7 healthy controls), lack of clarity on whether the self-injury was suicidal or non-suicidal, use of FA as opposed to GFA, and lack of a dimensional approach to gain a deeper understanding of this biological finding.

Using advanced dMRI (HARDI) methods, this study examined GFA in adolescents and young adults with NSSI versus healthy controls. We hypothesized that the NSSI group would show lower GFA than controls. More specifically, given the association between difficulties in self-regulation and NSSI [see $(16,17)$ for review], we anticipated that this would include lower GFA within white matter tracts from neural circuits that are known to be involved in self-regulation, such as the uncinate fasciculus and cingulum $(18,19)$. Further, within the NSSI group, we examined clinical correlates of GFA within the uncinate fasciculus and cingulum. We predicted that lower GFA would be associated with greater NSSI severity and with greater difficulties in self-regulation.

\section{METHOD}

\section{Participants}

Data were used from a recently completed study at the University of Minnesota (Cullen: 1R21MH094558), which was approved by the University of Minnesota Institutional Review Board. Female healthy controls (HC) and participants with NSSI aged 13-21 years were recruited using primarily community postings, clinic referrals, and online advertisements around the Minneapolis/ Saint Paul area. While the larger study was open to both males and females, only females were included for the present analyses as only one male participated. Inclusion criteria for the NSSI group included engaging in NSSI at least 4 times, with at least 1 episode occurring in the past month. Exclusion criteria for both groups was a history of bipolar, pervasive developmental or psychotic disorders, current pregnancy or breastfeeding, unstable medical illnesses, active suicidal intent, presence of MRIincompatible features, a positive urine drug screen, and intelligence quotient (IQ) of less than 80 as measured by the Wechsler Abbreviated Scale of Intelligence [WASI; (20)]. Additional exclusion criteria for HC included any history of self-injurious behavior (suicidal or non-suicidal) and any current or past DSM-IV psychiatric diagnoses. Interested participants contacted the research team via email or phone, which was followed by a phone screen to assess for basic inclusion and exclusion criteria.

Participants who appeared eligible via the phone screen were invited to participate in the initial screening visit. Participants with NSSI were offered three different options for study participation: (1) MRI study only; (2) treatment study only; or (3) both MRI and treatment study (MRI conducted both preand post-treatment). The treatment offered was an open label pilot study for the dietary supplement $\mathrm{N}$-acetylcysteine. Further description of this trial and its clinical results have been previously published (21). Participants in the HC group were only offered the option to participate in the MRI study. The present study includes data from participants who elected to complete the MRI-only study or the MRI and treatment study (using only the pre-treatment MRI data). Other neuroimaging data from this study (resting-state and task functional connectivity and psychophysiological interactions) have been published previously $(22,23)$. Once participants selected their desired study option, informed consent and assent (where applicable) were obtained. 


\section{Measures}

\section{Clinical Assessment}

Following informed consent and assent (as appropriate), all participants completed comprehensive diagnostic assessments, which were conducted by trained clinicians or graduate students or trainees under the supervision of a licensed psychologist or psychiatrist. Interviews were conducted separately with adolescents and parents, and included Kiddie Schedule for Affective Disorders and Schizophrenia-Present and Lifetime Version [K-SADS-PL; (24)] for participants under 18 years old and the Structured Clinical Interview for DSM-IV Axis I Disorders [SCID; $(25,26)]$ for participants 18 years old or older. For those under 18 years old, diagnoses were established via consensus between the adolescent and parent/guardian interviewers. Participants also completed the Beck Depression Inventory-II [BDI-II; (27)], which was used to control for depressive symptoms for within-NSSI group analyses.

\section{Non-Suicidal Self-Injury}

We assessed for NSSI using the self-report Inventory of Statements About Self-Injury [ISAS; (28)] and the clinicianadministered Deliberate Self-Harm Inventory [DSHI; (29)]. These two measures were used to provide a consensus on frequency and type of self-injury as well as duration of NSSI for each participant in the NSSI group. Average weekly cutting episodes were calculated by taking the consensus of lifetime cutting episodes from the ISAS and DSHI and dividing them by the estimated number of weeks the participant engaged in NSSI. We focused on cutting episodes for these analyses because cutting was the primary method of NSSI among all the NSSI participants. We used winsorization to reassign outliers on this variable to three standard deviations above the mean. Duration of NSSI was calculated by subtracting the age participants reported first engaging in NSSI from their current age.

\section{Self-Regulation Measures}

Measures of self-regulation included the Difficulties in Emotion Regulation Scale [DERS; (30)] and the Barratt Impulsiveness Scale [BIS; (31)]. The DERS includes six subscales in addition to a total score: Awareness, Clarity, Goals, Impulse, Nonacceptance, and Strategies. The factor structure of the DERS was initially found among adults (30) and has been replicated among adolescents (32). The DERS has internal consistency that ranges from acceptable to high across factors in both adolescents (average $\alpha=.81$ ) and adults (average $\alpha=.85$ ). The BIS includes three subscales in addition to a total score: Attentional, Motor, and Non-planning. The BIS total score has been found to have high internal consistency, with $\alpha$ ranging from.79 in substance-abuse patients and. 83 in general psychiatric patients (31). Total score and subscales from both the DERS and the BIS were used for analyses. In addition, we examined participants' $t$-scores on the Self-Harm subscale within the Borderline clinical scale (BOR-S) from the Personality Assessment Inventory (PAI) or Personality Assessment Inventory-Adolescent (PAI-A) for those under $18(33,34)$. The BOR-S scale is a measure of self-destructive and impulsive behavior in general and includes questions regarding behaviors at high-risk for negative consequences. While the PAI and PAI-A differ in length (PAI has 344 items and PAI-A has 242 items), the conversion of raw scores to $t$-scores allows for the two measures to be comparable. Both measures have shown high test-retest reliability with correlations of.80 or higher for all subscales of the PAI and an average correlation of.78 for the PAI-A. Additionally, both measures have demonstrated high internal consistency for the scales, with a median $\alpha$ of. 88 and average $\alpha$ of. 80 for the PAI and PAI-A respectively $(33,34)$.

\section{Neuroimaging Acquisition}

Following the first visit (consent and diagnostic/clinical assessment), participants completed an MRI scan at the Center for Magnetic Resonance Research at the University of Minnesota using a Siemens 3T TIM Trio scanner and a 32-channel receiveonly head coil. A pair of diffusion scans were acquired with identical parameters except with opposite phase encode directions (right to left and left to right) to estimate and correct for distortions. These scans were acquired using a multi-band EPI sequence with: 66 oblique axial slices; $2 \mathrm{~mm}$ isotropic voxel; 128 volumes with non-colinear diffusion directions and 17 volumes without diffusion weighting; flip angle $=90^{\circ} ; \mathrm{FOV}=212 \mathrm{~mm}$; multiband factor $=3$; b-value $=$ $1,500 \mathrm{~s} / \mathrm{mm}^{2} ; \mathrm{TR}=3,097 \mathrm{~ms} ; \mathrm{TE}=90.2 \mathrm{~ms}$.

\section{Statistical Analysis}

\section{Demographics and Clinical Data}

Demographic and clinical data were analyzed using IBM SPSS Version 24 (35). Descriptive variables of interest included age, IQ, scores on clinical measures of psychopathology, and current psychiatric diagnoses and medications.

\section{Diffusion MRI Preprocessing and Analysis}

Image processing was performed using software from the FSL toolkit (https://fsl.fmrib.ox.ac.uk/fsl/fslwiki). The topup tool from FSL was performed on the pair of dMRI scans (i.e., the right to left phase encode pair) from each participant to estimate the susceptibility induced off-resonance field. Each scan pair was then concatenated using $f_{\text {slmerge. Eddy-current and }}$ susceptibility-induced distortion corrections were completed using the Gaussian Process approach applied by eddy in FSL (36). We used the Brain Extraction Tool (BET) in FSL to complete brain extraction on the resulting data. Custom built tools created in MATLAB, as developed by Aganj and colleagues (37) based on the method presented by Assemlal, Tschumperlé, and Brun (12), were used to calculate ODF and create GFA maps for each individual.

Using the steps for Tract-Based Spatial Statistics (TBSS) in FSL (38), we performed nonlinear registration of the GFA maps into standard space, creation of mean GFA images and a white matter "skeleton" for each individual. This was followed by a projection of the GFA data from all subjects onto the mean GFA skeleton. For the purpose of examining brain-behavior correlates within the NSSI group, the JHU-ICBM-tracts-maxprob-thr0-1 $\mathrm{mm}$ atlas was used to create region of interest (ROI) masks for the right and left cingulum and uncinate fasciculus, which are 
both tracts known to be critical for self-regulation. The ROI masks were multiplied with the GFA mean skeleton to restrict the analyses to voxels within the skeleton and in the tracks of interest (see Figure 1). Finally, fslmeants was used to extract average GFA values within the skeleton portion of each of the four ROIs for all participants for clinical correlations.

\section{Statistical Analysis}

Group comparisons examining differences between NSSI and HC groups in GFA maps, while controlling for age, were completed using GLM modeling and the Threshold-Free Cluster Enhancement [TFCE; (39)] option with $p$-value $<.01$.

Within the NSSI group, we conducted correlations between GFA and clinical measures using partial Pearson's correlations controlling for age and IQ. To allow our analyses to be more specific to NSSI, as opposed to depressive symptoms, we included BDI scores as covariates. We performed correlations between GFA of the right and left uncinate fasciculus and scores from the 7 DERS scales, 4 BIS scales, and the BOR-S scale from the PAI/PAI-A. Given the total of 24 comparisons and our hypothesis that higher scores on these clinical measures will be associated with lower GFA values, we used a one-tailed $p$ value $<.002$ as our level of significance. We used the same method for the right and left cingulum including using a onetailed $p$-value $<.002$. We elected to use one-tailed $p$-values due to our a priori hypotheses that higher scores on clinical measures will correspond to lower GFA values.

We also explored whether there were any associations between NSSI severity (frequency and duration of NSSI) and
GFA in the cingulum and uncinate fasciculus. We used a onetailed $p$-value $<.0125$ in recognition of 4 comparisons for each NSSI frequency and duration.

\section{RESULTS}

\section{Demographic and Clinical Characteristics}

Overall, 29 NSSI and 22 HC completed all study procedures. After one subject was excluded due to poor dMRI data quality, data from 28 NSSI and $22 \mathrm{HC}$ participants were used for the final analyses. The number of NSSI participants who completed each of the clinical measures for this study varied. Further demographic and clinical characteristics for the sample can be found in Table 1.

\section{dMRI}

The whole brain group comparison analyses revealed several areas that showed significantly lower GFA in the NSSI group when compared to the HC group at a corrected $p<.01$ (corrected through permutation testing within TFCE as described in 39). In addition to the cingulum and uncinate fasciculus as predicted, these areas also included bilateral superior and inferior longitudinal fasciculi, anterior thalamic radiation, callosal body, and corticospinal tract. Figure 2 depicts the locations of these group differences.

Lower GFA of the left and right uncinate fasciculus was associated with higher total scores on the attentional subscale of the BIS. A correlation matrix can be found in Table 2 , which

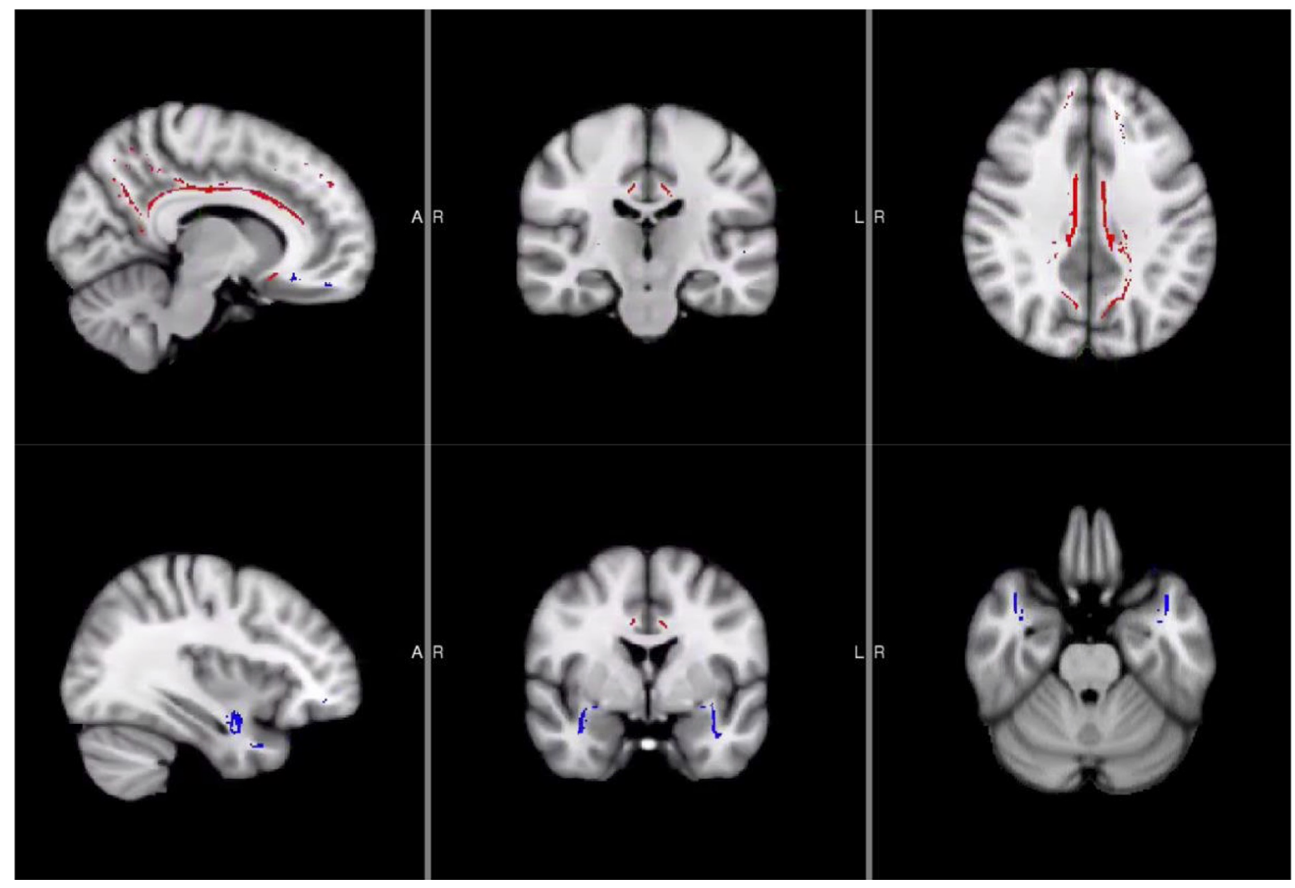

FIGURE 1 | Locations of Uncinate Fasciculus and Cingulum Masks. Areas in red were used for the cingulum masks while blue areas were used for the uncinate fasciculus masks. 
TABLE 1 | Participant demographics.

\begin{tabular}{|c|c|c|}
\hline $\begin{array}{l}\text { Demographic } \\
\text { Characteristics }\end{array}$ & NSSI $(n=28)$ & Controls $(n=22)$ \\
\hline Age (mean years $\pm \mathrm{SD}$ ) & $17.53 \pm 2.36$ & $17.69 \pm 2.26$ \\
\hline $\mathrm{IQ}($ mean $\pm \mathrm{SD})$ & $105.78 \pm 10.68(n=27)$ & $110.05 \pm 9.43(n=20)$ \\
\hline Right Handed - n (\%) & $24(89 \% ; n=27)$ & $19(100 \% ; n=19)$ \\
\hline \multicolumn{3}{|l|}{ Ethnicity - n (\%) } \\
\hline White & $26(93 \%)$ & 19 (86\%) \\
\hline African American & $1(4 \%)$ & $1(5 \%)$ \\
\hline Hispanic & $3(11 \%)$ & 0 \\
\hline Asian & 0 & $2(7 \%)$ \\
\hline Other & $1(4 \%)$ & 0 \\
\hline \multicolumn{3}{|l|}{ Clinical Measures } \\
\hline DERS Total** & $119.17 \pm 22.22(n=24)$ & $60.63 \pm 9.39(n=19)$ \\
\hline DERS Awareness ${ }^{* \star}$ & $20.63 \pm 6.35(n=24)$ & $12.26 \pm 3.35(n=19)$ \\
\hline DERS Clarity ${ }^{\star \star}$ & $16.69 \pm 4.30(n=26)$ & $8.48 \pm 1.66(n=21)$ \\
\hline DERS Goals* & $17.92 \pm 5.54(n=24)$ & $12.21 \pm 4.72(n=19)$ \\
\hline DERS Impulse $e^{\star \star}$ & $18.88 \pm 5.91(n=24)$ & $7.54 \pm 2.03(n=19)$ \\
\hline DERS Nonacceptance ${ }^{\star \star}$ & $18.92 \pm 6.21(n=24)$ & $9.11 \pm 2.89(n=19)$ \\
\hline DERS Strategies ${ }^{\star \star}$ & $26.29 \pm 5.65(n=24)$ & $10.95 \pm 3.10(n=19)$ \\
\hline BIS Total $\left.\right|^{\star \star}$ & $73.12 \pm 10.47(n=26)$ & $57.24 \pm 8.24(n=21)$ \\
\hline BIS Attentional ${ }^{\star \star}$ & $21.00 \pm 3.71(n=26)$ & $15.05 \pm 3.07(n=21)$ \\
\hline BIS Motor ${ }^{\star \star}$ & $24.31 \pm 5.63(n=26)$ & $18.76 \pm 3.86(n=21)$ \\
\hline BIS Nonplanning ${ }^{\star}$ & $27.81 \pm 3.67(n=26)$ & $23.43 \pm 4.91(n=21)$ \\
\hline PAI/PAI-A Borderline-Self & $64.88 \pm 15.06(n=25)$ & $44.95 \pm 11.49(n=19)$ \\
\hline Harm Subscale ${ }^{\star \star}$ & & \\
\hline \multicolumn{3}{|l|}{ NSSI Characteristics } \\
\hline Age of first NSSI (mean \pm SD) & $11.96 \pm 3.03(n=27)$ & \\
\hline $\begin{array}{l}\text { Lifetime Cutting Episodes } \\
\text { (mean } \pm \mathrm{SD})\end{array}$ & $131.11 \pm 195.43$ & \\
\hline $\begin{array}{l}\text { Estimated Cutting Episodes } \\
\text { per Week (mean } \pm \mathrm{SD})^{\circ}\end{array}$ & $0.75 \pm 1.16$ & \\
\hline $\begin{array}{l}\text { Duration (years) of NSSI } \\
(\text { mean } \pm \mathrm{SD})^{d}\end{array}$ & $5.43 \pm 3.85(n=27)$ & \\
\hline \multicolumn{3}{|l|}{ Current Diagnoses - n (\%) } \\
\hline Major Depressive Disorder & $16(57 \%)$ & \\
\hline Depressive Disorder NOS & $5(18 \%)$ & \\
\hline Generalized Anxiety Disorder & $8(29 \%)$ & \\
\hline Anxiety Disorder NOS & $2(7 \%)$ & \\
\hline Social Phobia & $1(4 \%)$ & \\
\hline Specific Phobia & $3(11 \%)$ & \\
\hline Panic Disorder & $3(11 \%)$ & \\
\hline Post-Traumatic Stress & $5(18 \%)$ & \\
\hline \multicolumn{3}{|l|}{ Disorder } \\
\hline Obsessive Compulsive & $2(7 \%)$ & \\
\hline \multicolumn{3}{|l|}{ Disorder } \\
\hline Eating Disorder NOS & $1(4 \%)$ & \\
\hline ADHD & $2(7 \%)$ & \\
\hline Alcohol Dependence & $2(7 \%)$ & \\
\hline No Current Disorder & $5(18 \%)$ & \\
\hline \multicolumn{3}{|l|}{ Medications } \\
\hline Currently Medicated & $12(43 \%)$ & \\
\hline Antidepressants & $9(32 \%)$ & \\
\hline Stimulants & $2(7 \%)$ & \\
\hline Antipsychotics & $1(4 \%)$ & \\
\hline Antianxiety/Benzodiazepines & $4(14 \%)$ & \\
\hline Other Psychotropics & $1(4 \%)$ & \\
\hline $\begin{array}{l}{ }^{*} p<.005 . \\
{ }^{* *} p<.001 . \\
{ }^{a} \text { Post-hoc analyses indicated tha } \\
{ }^{b} \text { Participants were able to endors } \\
{ }^{c} \text { Consensus between ISAS and } \\
\text { cutting episodes per week. These } \\
{ }^{d} \text { Duration of NSSI calculated by s }\end{array}$ & $\begin{array}{l}\text { differing handedness did no } \\
\text { DSHI was calculated to dete } \\
\text { are pre-Winsorized scores. } \\
\text { ubtracting age of first NSSI }\end{array}$ & $\begin{array}{l}\text { t affect study findings. } \\
\text { thnicity. } \\
\text { ermine average number of } \\
\text { from current age. }\end{array}$ \\
\hline
\end{tabular}

includes comparisons that were significant at an uncorrected $p<.05$. For the left and right cingulum, there were no significant associations with measures of self-regulation (DERS and BIS). A correlation matrix can be found in Table 3. Finally, lower GFA of the left cingulum was associated with a longer duration of NSSI. There were no significant correlations between other severity indices (e.g., average number of episodes) and GFA. These in addition to results from other comparisons can be found in the correlation matrix in Table 4.

\section{DISCUSSION}

In this study of white matter microstructure in adolescents and young adults with NSSI, we report extensive group differences in GFA between females with NSSI and healthy controls in the uncinate fasciculus, cingulum, and several other white matter tracts throughout the brain; which is consistent with our hypotheses. This suggests NSSI may be associated with a broad array of white matter disorganization that extends beyond the scope of the present study. However, future research investigating the potential functional meaning of these other white matter deficits may be beneficial. We also report associations between GFA in the uncinate fasciculus and cingulum and clinical characteristics (measures of selfregulation and NSSI characteristics). Specifically, higher levels of attentional impulsivity; characterized by racing thoughts, difficulty with focus, and intrusive thoughts (31); were associated with lower GFA in the left uncinate fasciculus. Additionally, longer duration of NSSI was associated with lower GFA in the left cingulum. These findings suggest that among participants with NSSI, greater psychopathology in key domains (impulsivity, severity of self-harm) may be explained by greater disorganization in key frontolimbic white matter tracts in this still-developing population.

\section{Group Differences in Whole Brain GFA Between NSSI and HC}

Adolescents and young adults with NSSI showed lower GFA compared to the HC group in the uncinate fasciculus and cingulum, which is consistent with our hypotheses. In addition, the NSSI group showed lower GFA in several other areas including the inferior and superior longitudinal fasciculi, callosal body, forceps major and minor, anterior thalamic radiation, and corticospinal tract. To our knowledge, only one other study has examined differences in structural connectivity between those with and without self-injury and found compromised white matter microstructure within the frontal lobe in adults with BPD (15). The present study differs from this previous study as it examines adolescents, examines NSSI across diagnoses, uses a larger sample size, employs methods that result in a potentially more accurate scalar measure of white matter integrity (GFA), and also investigates NSSI more explicitly as it is unclear whether the previous study included suicidal self-injury. 


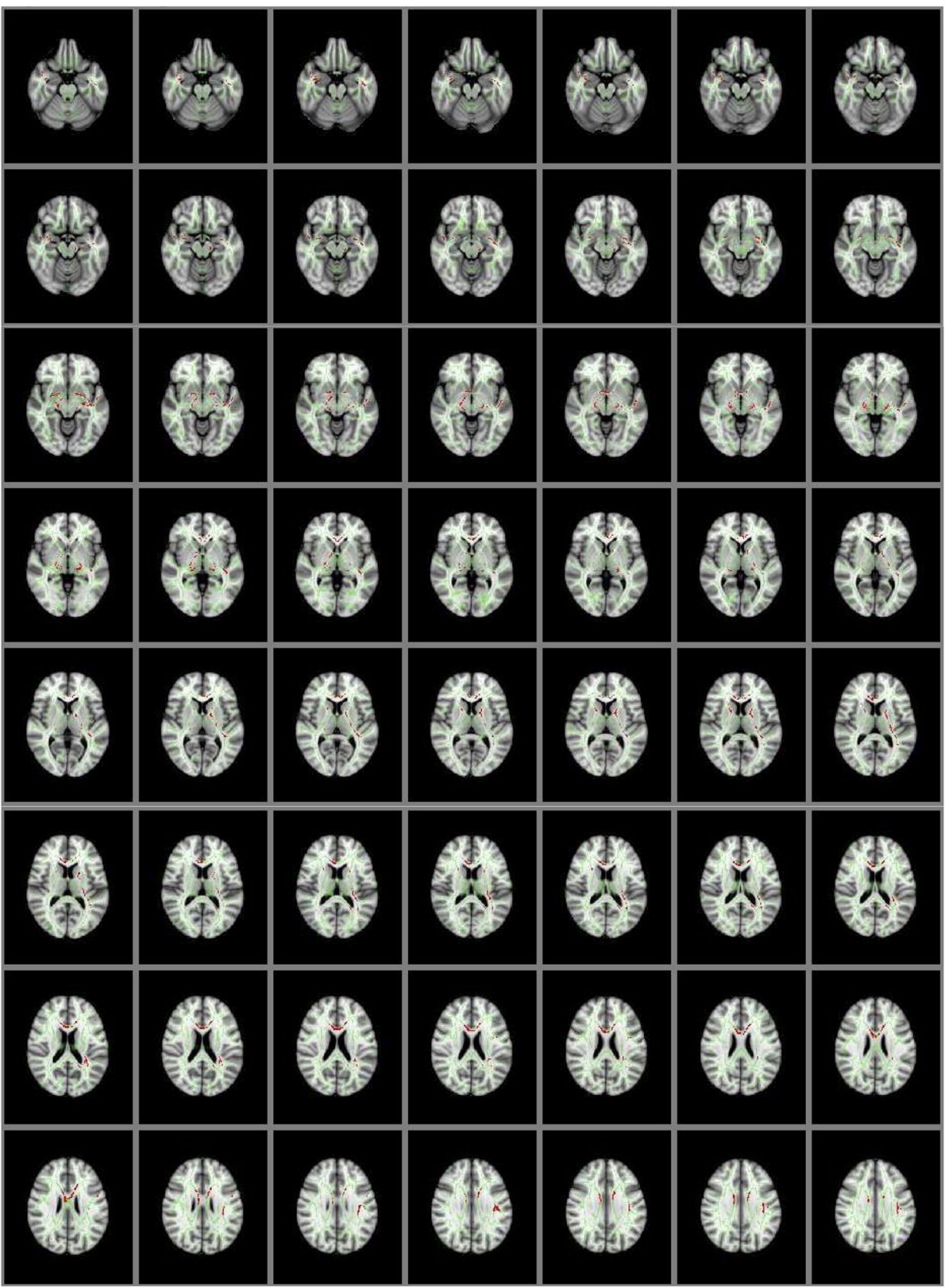

FIGURE 2 | Group Differences in GFA: Controls > NSSI. White matter tracts in red show where controls have significantly greater GFA than NSSI. This is overlaid on the mean GFA skeleton (light green). Findings were significant at $p<.01$.

\section{ROI-Specific GFA Associations With Clinical Measures}

We also examined the association between clinical measures and GFA within the NSSI group while controlling for age, IQ, and BDI-II scores. Lower GFA within the uncinate fasciculus, which serves brain regions implicated in self-regulation, was significantly associated with higher scores on the attentional subscale of the BIS. Given the role the uncinate fasciculus plays in serving as a connection between subcortical structures and frontal regulatory regions, the present findings suggest that those who experience racing and intrusive thoughts or difficulties with focusing on tasks may show compromised white matter organization within this tract. Decreased FA in the uncinate fasciculus has been associated with $\operatorname{BPD}(40,41)$, emotion dysregulation disorders (42), and suicide attempts (43-46). While the relationship between NSSI and impulsivity remains 
TABLE 2 | Self-regulation and uncinate fasciculus correlations.

\begin{tabular}{|c|c|c|c|}
\hline \multicolumn{2}{|c|}{ Control Variables: Age \& BDI \& IQ } & \multicolumn{2}{|c|}{ Correlations } \\
\hline & & $\begin{array}{l}\text { Left Uncinate } \\
\text { Fasciculus }\end{array}$ & $\begin{array}{l}\text { Right Uncinate } \\
\text { Fasciculus }\end{array}$ \\
\hline \multirow[t]{3}{*}{ BOR-S } & Correlation & -.341 & -.329 \\
\hline & Significance (1-tailed) & .051 & .062 \\
\hline & df & 21 & 21 \\
\hline BIS & Correlation & -.668 & -.573 \\
\hline \multirow[t]{2}{*}{ Attentional } & Significance (1-tailed) & $<.001^{\star}$ & $.002^{*}$ \\
\hline & df & 22 & 22 \\
\hline \multirow[t]{3}{*}{ BIS Motor } & Correlation & -.383 & -.374 \\
\hline & Significance (1-tailed) & .032 & .036 \\
\hline & df & 22 & 22 \\
\hline BIS & Correlation & -.306 & -.137 \\
\hline \multirow[t]{2}{*}{ Nonplanning } & Significance (1-tailed) & .073 & .262 \\
\hline & df & 22 & 22 \\
\hline \multirow[t]{3}{*}{ BIS Total } & Correlation & -.550 & -.447 \\
\hline & Significance (1-tailed) & .003 & .014 \\
\hline & df & 22 & 22 \\
\hline DERS & Correlation & .109 & .066 \\
\hline \multirow[t]{2}{*}{ Nonaccept } & Significance (1-tailed) & .315 & .385 \\
\hline & df & 20 & 20 \\
\hline \multirow[t]{3}{*}{ DERS Goals } & Correlation & -.348 & -.300 \\
\hline & Significance (1-tailed) & .056 & .088 \\
\hline & df & 20 & 20 \\
\hline DERS & Correlation & -.038 & -.066 \\
\hline \multirow[t]{2}{*}{ Impulse } & Significance (1-tailed) & .433 & .386 \\
\hline & df & 20 & 20 \\
\hline DERS & Correlation & .360 & .119 \\
\hline \multirow[t]{2}{*}{ Awareness } & Significance (1-tailed) & .050 & .298 \\
\hline & df & 20 & 20 \\
\hline DERS & Correlation & .064 & .074 \\
\hline \multirow[t]{2}{*}{ Strategies } & Significance (1-tailed) & .389 & .371 \\
\hline & df & 20 & 20 \\
\hline \multirow[t]{3}{*}{ DERS Clarity } & Correlation & .162 & .020 \\
\hline & Significance (1-tailed) & .224 & .463 \\
\hline & df & 22 & 22 \\
\hline \multirow[t]{3}{*}{ DERS Total } & Correlation & .079 & -.037 \\
\hline & Significance (1-tailed) & .363 & .435 \\
\hline & df & 20 & 20 \\
\hline
\end{tabular}

*Meets criteria for significance based on corrected p-value $<.002$.

controversial, a recent review provides helpful insight regarding the complexities of this relationship. Lockwood and colleagues (17) highlight that mood-dependent impulsivity, such as that measured by Negative Urgency in the UPPS Impulsive Behavior Scale (47), predisposes an individual to begin engaging in NSSI; while higher scores on more cognitively-related facets of impulsivity were more reflective of recent NSSI, and thus may serve to maintain the behavior. This is consistent with the relationship between BIS scores and GFA within our sample of NSSI participants as they had been engaging in recent selfinjury. However, it is necessary to further elaborate on these relationships by also incorporating measures investigating mood-dependent impulsivity.

We also found that longer duration of NSSI was associated with lower GFA within the left and right cingulum. Because we controlled for current age in these analyses, this finding suggests that the impaired white matter integrity of this region among those with NSSI may be the result of a cumulative effect over time. However, it is also important to consider the high
TABLE 3 | Self-regulation and cingulum correlations.

\begin{tabular}{|c|c|c|c|}
\hline \multicolumn{2}{|c|}{ Control Variables: Age \& BDI \& IQ } & \multicolumn{2}{|c|}{ Correlations } \\
\hline & & Left Cingulum & Right Cingulum \\
\hline \multirow[t]{3}{*}{ BOR-S } & Correlation & -.442 & -.507 \\
\hline & Significance (1-tailed) & .017 & .007 \\
\hline & $\mathrm{df}$ & 21 & 21 \\
\hline \multirow[t]{3}{*}{ BIS Attentional } & Correlation & -.392 & -.455 \\
\hline & Significance (1-tailed) & .029 & .013 \\
\hline & df & 22 & 22 \\
\hline \multirow[t]{3}{*}{ BIS Motor } & Correlation & -.411 & -.502 \\
\hline & Significance (1-tailed) & .023 & .006 \\
\hline & df & 22 & 22 \\
\hline \multirow[t]{3}{*}{ BIS Nonplanning } & Correlation & -.223 & -.333 \\
\hline & Significance (1-tailed) & .147 & .056 \\
\hline & df & 22 & 22 \\
\hline \multirow[t]{3}{*}{ BIS Total } & Correlation & -.436 & -.547 \\
\hline & Significance (1-tailed) & .017 & .003 \\
\hline & df & 22 & 22 \\
\hline \multirow[t]{3}{*}{ DERS Nonaccept } & Correlation & .066 & -.048 \\
\hline & Significance (1-tailed) & .384 & .417 \\
\hline & $\mathrm{df}$ & 20 & 20 \\
\hline \multirow[t]{3}{*}{ DERS Goals } & Correlation & .053 & .019 \\
\hline & Significance (1-tailed) & .408 & .467 \\
\hline & df & 20 & 20 \\
\hline \multirow[t]{3}{*}{ DERS Impulse } & Correlation & -.330 & -.268 \\
\hline & Significance (1-tailed) & .067 & .114 \\
\hline & df & 20 & 20 \\
\hline \multirow[t]{3}{*}{ DERS Awareness } & Correlation & -.179 & -.033 \\
\hline & Significance (1-tailed) & .212 & .332 \\
\hline & df & 20 & 20 \\
\hline \multirow[t]{3}{*}{ DERS Strategies } & Correlation & .358 & .279 \\
\hline & Significance (1-tailed) & .051 & .104 \\
\hline & df & 20 & 20 \\
\hline \multirow[t]{3}{*}{ DERS Clarity } & Correlation & -.277 & -.235 \\
\hline & Significance (1-tailed) & .095 & .135 \\
\hline & df & 22 & 22 \\
\hline \multirow[t]{3}{*}{ DERS Total } & Correlation & -.076 & -.077 \\
\hline & Significance (1-tailed) & .368 & .367 \\
\hline & df & 20 & 20 \\
\hline
\end{tabular}

TABLE 4 | Duration of non-suicidal self-injury (NSSI) and cutting frequency and GFA correlations.

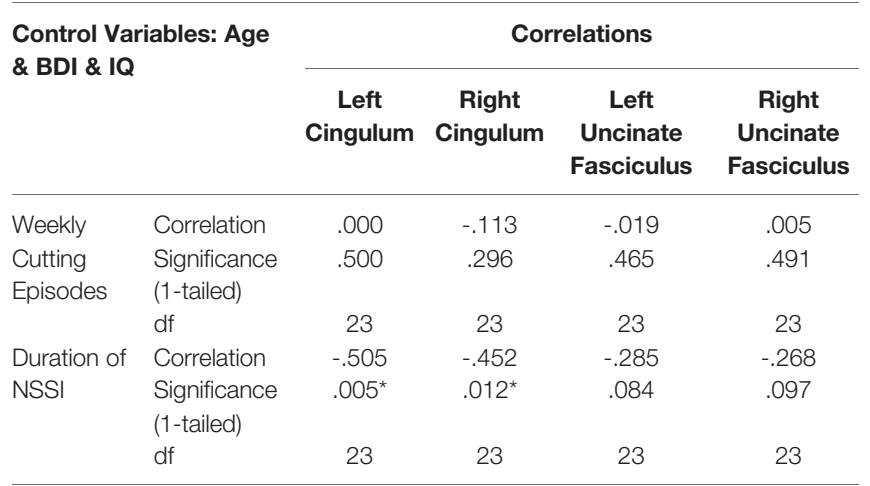

*Meets criteria for significance based on corrected p-value <.0125.

likelihood that any existing psychopathology had developed concurrently, or had already existed, around the time of first NSSI episode. Although some studies have reported null findings regarding differences in FA of the cingulum between psychiatric 
samples and controls $(48,49)$, a meta-analysis of adolescents with major depressive disorder (MDD) found that overall, those with depression had decreased FA within this region (50). Our finding highlights the importance of early intervention and the utility it may have in preventing aberrant, or restoring normal, neurodevelopmental trajectories. Further, given the number of functions in which the cingulum plays a role, including emotion processing, pain, and executive functioning (18), it is imperative that there is continued investigation into this possible disruption as it may lead to entrenchment of maladaptive behaviors and poorer prognosis.

\section{Strengths and Limitations}

This study represents a significant advancement of existing NSSI work as it used an approach to the dMRI data that lessens the impact of crossing fibers when compared to dMRI methods used in previous studies. Unlike many previous studies of NSSI, which investigate the behavior in the context of a specific diagnosis, the present study examines the neural circuitry of NSSI across diagnoses. As a strength, the presence of varying types and levels of psychopathology seen in this study is consistent with what has been found in larger studies of NSSI $(51,52)$ and may reflect a more representative sample of those with NSSI. However, taking a diagnostic-independent approach also poses a limitation as it is difficult to determine whether findings are specific to NSSI or to psychopathology more broadly. We aimed to limit this influence by controlling for depression symptoms when performing our withinNSSI group analyses, as depressive disorders were the most common diagnoses in our sample. However, it would be beneficial for future research to incorporate a psychiatric control group that is matched to the NSSI group on diagnosis and severity level/level of impairment. Considerations of external validity should be considered given that the present study consisted of only females and primarily older adolescents/young adults. Further, given that there was an experimental intervention offered as part of the larger study, participants may be more likely to be willing to disclose NSSI and be treatment-seeking. Additional limitations include the inability to generalize to males, the likelihood that the present sample was ready to seek treatment, and the sample consisting of mainly older adolescents.

The cross-sectional design of this study is also a limitation, particularly when interpreting the association between longer duration of NSSI and lower GFA. While we do believe this finding supports a treatment approach with an earlier intervention and prevention, it is still imperative to fully explore whether this white matter disruption was present before or after NSSI onset. Longitudinal designs may also help in developing our understanding of the mechanisms of change with successful intervention strategies, which may then be used to target neurobiologically-based deficits associated with NSSI.

It is possible that these white matter anomalies are common across a range of psychopathology. Relying on FA as opposed to GFA, studies examining dMRI have found compromised white matter microstructure associated with psychopathology within these tracts including adults with MDD (53), PTSD (54), and childhood adversity (55), and adolescents with BPD (41).
Additionally, a meta-analysis of FA in emotional disorders (MDD, bipolar disorder, social anxiety disorder, obsessivecompulsive disorder, and PTSD) found significantly lower FA compared to healthy controls in the forceps minor, uncinate fasciculus, anterior thalamic radiation, and superior longitudinal fasciculus (56). Given that most individuals in the NSSI group in the present study had a current diagnosis of emotional disorders, such as those included in the meta-analysis by Jenkins and colleagues (56), the widespread findings of lower GFA in this group may reflect overall psychopathology present in this sample. With this in mind, future research examining GFA in adolescents with NSSI should consider incorporating a psychiatric control group to allow for greater specificity in understanding the aberrations that are unique to NSSI.

Finally, our sample size, while much larger than the previous study examining self-injury and white matter integrity, limits our ability to conduct correlational analyses given the number of comparisons and limited power. While there are other clinical measures that may be of interest to explore within this sample, such as identity disturbance and interpersonal sensitivity, we limited our analyses to these specific constructs of self-regulation given the existing literature in NSSI. In the future, larger studies will be better suited to more fully examine other constructs implicated in NSSI.

\section{CONCLUSION}

This is among one of the first studies to provide evidence for the role of compromised white matter organization and its relationship to clinical measures among adolescents and young adults with NSSI. Categorical analyses revealed that compared to healthy controls, the NSSI group exhibited widespread white matter disruption, consistent with other forms of psychopathology including depression and suicide. Dimensional analyses revealed that among those with NSSI, levels of attentional impulsivity and the duration of NSSI within the NSSI group were associated with lower white matter integrity in the uncinate fasciculus and cingulum. Clinically, these findings provide some insight as to how interventions that focus on self-regulation, such as dialectical behavior therapy (DBT), have shown success in treating NSSI (57). Additionally, the association between duration of NSSI and GFA provides further support for the importance of early intervention in hopes to restore healthy neurodevelopmental trajectories. However, longitudinal research is needed to understand when and how these white matter abnormalities develop, whether they predispose adolescents to developing maladaptive behaviors such as NSSI or if they emerge later in the disease course. This knowledge may then contribute to the foundation of more effective and targeted interventions.

\section{DATA AVAILABILITY STATEMENT}

The datasets generated for this study are available on request to the corresponding author. 


\section{ETHICS STATEMENT}

The studies involving human participants were reviewed and approved by University of Minnesota Institutional Review Board. Written informed consent to participate in this study was provided by the participants' legal guardian/next of kin.

\section{AUTHOR CONTRIBUTIONS}

MW conceived and designed the analyses, collected the data, performed analyses, and wrote the manuscript. BM conceived and designed the analyses, collected the data, performed analyses, and contributed to manuscript writing. BK-D assisted in study conception and design, data collection, and contributed to manuscript writing. EB contributed to manuscript writing and data collection. MF contributed biostatistical guidance. DH contributed to manuscript writing. KL contributed to study conception and design. KC contributed funding, conception and design, data collection, analyses, and manuscript writing.

\section{FUNDING}

This study was funded by the National Institute of Mental Health grant 1R21MH094558 (KC), the University of

\section{REFERENCES}

1. Horwitz AG, Czyz EK, King CA. Predicting future suicide attempts among adolescent and emerging adult psychiatric emergency patients. J Clin Child Adolescent Psychol (2014) 53:1-11. doi: 10.1080/15374416.2014.910789

2. Tang J, Yu Y, Wu Y, Du Y, Ma Y, Zhu H, et al. Association between nonsuicidal self-injuries and suicide attempts in Chinese adolescents and college students: a cross-section study. PloS One (2011) 6(4):e17977. doi: 10.1371/ journal.pone.0017977

3. Victor SE, Klonsky ED. Correlates of suicide attempts among self-injurers: a meta-analysis. Clin Psychol Rev (2014) 34(4):282-97. doi: 10.1016/ j.cpr.2014.03.005

4. Giedd JN, Blumenthal J, Jeffries NO, Castellanos FX, Liu H, Zijdenbos A, et al. Brain development during childhood and adolescence: a longitudinal MRI study. Nat Neurosci (1999) 2(10):861-3. doi: 10.1038/13158

5. Jacobson CM, Gould M. The epidemiology and phenomenology of non-suicidal self-injurious behavior among adolescents: a critical review of the literature. Arch Suicide Res (2007) 11(2):129-47. doi: 10.1080/ 13811110701247602

6. Lenroot RK, Giedd JN. Brain development in children and adolescents: insights from anatomical magnetic resonance imaging. Neurosci Biobehav Rev (2006) 30(6):718-29. doi: 10.1016/j.neubiorev.2006.06.001

7. Turken AU, Whitfield-Gabrieli S, Bammer R, Baldo JV, Dronkers NF, Gabrieli JDE. Cognitive processing speed and the structure of white matter pathways: convergent evidence from normal variation and lesion studies. NeuroImage (2008) 42(2):1032-44. doi: 10.1016/j.neuroimage.2008.03.057

8. Beaulieu C. The basis of anisotropic water diffusion in the nervous system - a technical review. NMR Biomed (2002) 15(7-8):435-55. doi: 10.1002/nbm.782

9. O'Donnell LJ, Westin CF. An introduction to diffusion tensor image analysis. Neurosurg Clin North Am (2011) 22:185-96. doi: 10.1016/j.nec. 2010.12.004

10. Descoteaux M, Angelino E, Fitzgibbons S, Deriche R. Regularized, fast, and robust analytical Q-ball imaging. Magnetic Resonance Med (2007) 58(3):497510. doi: $10.1002 / \mathrm{mrm} .21277$

11. Tuch DS. Q-ball imaging. Magnetic Resonance Med (2004) 52(6):1358-72. doi: $10.1002 / \mathrm{mrm} .20279$
Minnesota Academic Health Center Faculty Research Development Grant Program \#11.12, the University of Minnesota Clinical and Translational Science Award UL1TR000114, and the National Institute of Biomedical Imaging and Bioengineering P41 EB015894 granted to the Center for Magnetic Resonance Research. MW's time on this study was funded by the University of Minnesota's Doctoral Dissertation Fellowship.

\section{ACKNOWLEDGMENTS}

First and foremost, the authors would like to thank the participants and their families who dedicated their time and effort to our research. We would also like to thank Drs. Nicholas Davenport and Christophe Lenglet for providing us with and allowing us to use their customized dMRI tools for calculating GFA. Additionally, we greatly appreciate the assistance of our volunteers, students, and staff who helped with recruitment, coordination, and data collection and management. Some findings from this study have been previously presented at a symposium for the 2018 International Society for the Study of Self-Injury conference. Additionally, this study also served as partial fulfillment of MW's doctoral dissertation.

12. Assemlal H-E, Tschumperle D, Brun L. (2007). Fiber Tracking on HARDI Data using Robust ODF Fields. 2007 IEEE International Conference on Image Processing, III-133-III-136. doi: 10.1109/ICIP.2007.4379264.

13. Pierpaoli C, Barnett A, Pajevic S, Chen R, Penix LR, Virta A, et al. Water diffusion changes in Wallerian degeneration and their dependence on white matter architecture. NeuroImage (2001) 13(6 Pt 1):1174-85. doi: 10.1006/nimg.2001.0765

14. Wiegell MR, Larsson HB, Wedeen VJ. Fiber crossing in human brain depicted with diffusion tensor MR imaging. Radiology (2000) 217(3):897-903. doi: 10.1148/radiology.217.3.r00nv43897

15. Grant JE, Correia S, Brennan-Krohn T, Malloy PF, Laidlaw DH, Schulz SC. Frontal white matter integrity in borderline personality disorder with selfinjurious behavior. J Neuropsychiat Clin Neurosci (2007) 19(4):383-90. doi: 10.1176/appi.neuropsych.19.4.383

16. Cullen KR, Westlund MK, LaRiviere LL, Klimes-Dougan B. An adolescent with nonsuicidal self-injury: a case and discussion of neurobiological research on emotion regulation. Am J Psychiatry (2013) 170(8):828-31. doi: 10.1176/ appi.ajp.2013.12121598

17. Lockwood J, Daley D, Townsend E, Sayal K. Impulsivity and self-harm in adolescence: a systematic review. Eur Child Adolescent Psychiatry (2017) 26 (4):387-402. doi: 10.1007/s00787-016-0915-5

18. Bubb EJ, Metzler-Baddeley C, Aggleton JP. The cingulum bundle: Anatomy, function, and dysfunction. Neurosci Biobehav Rev (2018) 92:104-27. doi: 10.1016/J.NEUBIOREV.2018.05.008

19. Von Der Heide RJ, Skipper LM, Klobusicky E, Olson IR. Dissecting the uncinate fasciculus: disorders, controversies and a hypothesis. Brain J Neurol (2013) 136(Pt 6):1692-707. doi: 10.1093/brain/awt094

20. Wechsler D. Wechsler Abbreviated Scale of Intelligence. The Psychological Corporation: New York, NY: Harcourt Brace \& Company (1999).

21. Cullen KR, Klimes-Dougan B, Westlund Schreiner M, Carstedt P, Marka N, Nelson K, et al. N-Acetylcysteine for nonsuicidal self-injurious behavior in adolescents: an open-label pilot study. J Child Adolescent Psychopharmacol (2018) 28(2):136-44. doi: 10.1089/cap.2017.0032

22. Demers LA, Schreiner MW, Hunt RH, Mueller BA, Klimes-Dougan B, Thomas KM, et al. Alexithymia is associated with neural reactivity to masked emotional faces in adolescents who self-harm. J Affective Disord (2019) 249:253-61. doi: 10.1016/J.JAD.2019.02.038 
23. Westlund Schreiner M, Klimes-Dougan B, Mueller BA, Eberly LE, Reigstad $\mathrm{KM}$, Carstedt PA, et al. Multi-modal neuroimaging of adolescents with nonsuicidal self-injury: Amygdala functional connectivity. I Affective Disord (2017) 221:47-55. doi: 10.1016/j.jad.2017.06.004

24. Kaufman J, Birmaher B, Brent D, Rao U, Flynn C, Moreci P, et al. Schedule for affective disorders and schizophrenia for school-age children-present and lifetime version (K-SADS-PL): initial reliability and validity data. J Am Acad Child Adolescent Psychiatry (1997) 36(7):980-8. doi: 10.1097/00004583199707000-00021

25. First MB, Spitzer RL, Gibbon M, Williams JBW. Structured Clinical Interview for DSM-IV-TR Axis I Disorders, Research Version, Non-patient Edition. (SCID-I/NP). New York, NY: Biometrics Research (2002a).

26. First MB, Spitzer RL, Gibbon M, Williams JBW. Structured Clinical Interview for DSM-IV-TR Axis I Disorders, Research Version, Patient Edition. (SCID-I/ P). Biometrics Research: New York, NY (2002b).

27. Beck AT, Steer RA, Brown GK. Manual for the Beck Depression Inventory-II. San Antonio, TX: Psychological Corporation (1996).

28. Glenn CR, Klonsky ED. One-year test-retest reliability of the inventory of statements about self-injury (ISAS). Assessment (2011) 18(3):375-8. doi: $10.1177 / 1073191111411669$

29. Gratz KL. Measurement of deliberate self-harm: preliminary data on the deliberate self-harm inventory. J Psychopathol Behav Assessment (2001) 23 (4):253-63. doi: 10.1023/A:1012779403943

30. Gratz KL, Roemer L. Multidimensional assessment of emotion regulation and dysregulation: development, factor structure, and initial validation of the difficulties in emotion regulation scale. J Psychopathol Behav Assessment (2004) 26(1):41-54. doi: 10.1023/B:JOBA.0000007455.08539.94

31. Patton JH, Stanford MS, Barratt ES. Factor structure of the Barratt impulsiveness scale. J Clin Psychol (1995) 51(6):768-74. doi: 10.1002/10974679(199511)51:6<768::AID-JCLP2270510607>3.0.CO;2-1

32. Neumann A, van Lier PAC, Gratz KL, Koot HM. Multidimensional assessment of emotion regulation difficulties in adolescents using the difficulties in emotion regulation scale. Assessment (2010) 17(1):138-49. doi: $10.1177 / 1073191109349579$

33. Morey LC. Personality Assessment Inventory. 2nd edn. Psychological Assessment Resources: Lutz, FL (2007b).

34. Morey LC. Personality Assessment Inventory-Adolescent. Psychological Assessment Resources: Lutz, FL (2007a).

35. IBM. IBM SPSS Statistics for Mac. Armonk, NY: IBM Corp (2016).

36. Andersson JLR, Sotiropoulos SN. An integrated approach to correction for off-resonance effects and subject movement in diffusion MR imaging. NeuroImage (2016) 125:1063-78. doi: 10.1016/j.neuroimage.2015.10.019

37. Aganj I, Lenglet C, Sapiro G, Yacoub E, Ugurbil K, Harel N. Reconstruction of the orientation distribution function in single- and multiple-shell q-ball imaging within constant solid angle. Magnetic Resonance Med (2010) 64 (2):554-66. doi: 10.1002/mrm.22365

38. Smith SM, Jenkinson M, Johansen-Berg H, Rueckert D, Nichols TE, Mackay CE, et al. Tract-based spatial statistics: Voxelwise analysis of multi-subject diffusion data. NeuroImage (2006) 31(4):1487-505. doi: 10.1016/j.neuroimage.2006.02.024

39. Smith SM, Nichols TE. Threshold-free cluster enhancement: Addressing problems of smoothing, threshold dependence and localisation in cluster inference. NeuroImage (2009) 44(1):83-98. doi: 10.1016/j.neuroimage.2008.03.061

40. Lischke A, Herpertz SC, Berger C, Domes G, Gamer M. Divergent effects of oxytocin on (para-)limbic reactivity to emotional and neutral scenes in females with and without borderline personality disorder. Social Cogn Affective Neurosci (2017) 12(11):1783-92. doi: 10.1093/scan/nsx107

41. New AS, Carpenter DM, Perez-Rodriguez MM, Ripoll LH, Avedon J, Patil U, et al. Developmental differences in diffusion tensor imaging parameters in borderline personality disorder. J Psychiatr Res (2013) 47(8):1101-9. doi: 10.1016/j.jpsychires.2013.03.021

42. Versace A, Acuff H, Bertocci MA, Bebko G, Almeida JRC, Perlman SB, et al. White matter structure in youth with behavioral and emotional dysregulation disorders. JAMA Psychiatry (2015) 72(4):367. doi: 10.1001/jamapsychiatry. 2014.2170

43. Jia Z, Huang X, Wu Q, Zhang T, Lui S, Zhang J, et al. High-field magnetic resonance imaging of suicidality in patients with major depressive disorder.
Am J Psychiatry (2010) 167(11):1381-90. doi: 10.1176/appi.ajp.2010. 09101513

44. Johnston JAY, Wang F, Liu J, Blond BN, Wallace A, Liu J, et al. Multimodal neuroimaging of frontolimbic structure and function associated with suicide attempts in adolescents and young adults with bipolar disorder. Am J Psychiatry (2017) 174(7):667-75. doi: 10.1176/appi.ajp.2016.15050652

45. Mahon K, Burdick KE, Wu J, Ardekani BA, Szeszko PR. Relationship between suicidality and impulsivity in bipolar I disorder: a diffusion tensor imaging study. Bipolar Disord (2012) 14(1):80-9. doi: 10.1111/j.1399-5618.2012.00984.x

46. Olvet DM, Peruzzo D, Thapa-Chhetry B, Sublette ME, Sullivan GM, Oquendo MA, et al. A diffusion tensor imaging study of suicide attempters. J Psychiatr Res (2014) 51:60-7. doi: 10.1016/j.jpsychires.2014.01.002

47. Whiteside SP, Lynam DR. The five factor model and impulsivity: using a structural model of personality to understand impulsivity. Personality Individual Differences (2001) 30(4):669-89. doi: 10.1016/S0191-8869(00) 00064-7

48. LeWinn KZ, Connolly CG, Wu J, Drahos M, Hoeft F, Ho TC, et al. White matter correlates of adolescent depression: structural evidence for frontolimbic disconnectivity. J Am Acad Child Adolescent Psychiatry (2014) 53(8):899-909.e7. doi: 10.1016/j.jaac.2014.04.021

49. Lischke A, Domin M, Freyberger HJ, Grabe HJ, Mentel R, Bernheim D, et al. Structural alterations in white-matter tracts connecting (para-)limbic and prefrontal brain regions in borderline personality disorder. Psycholog Med (2015) 45(15):3171-80. doi: 10.1017/S0033291715001142

50. Lichenstein SD, Verstynen T, Forbes EE. Adolescent brain development and depression: A case for the importance of connectivity of the anterior cingulate cortex. Neurosci Biobehav Rev (2016) 70:271-87. doi: 10.1016/j.neubiorev. 2016.07.024

51. In-Albon T, Ruf C, Schmid M. Proposed diagnostic criteria for the DSM-5 of nonsuicidal self-injury in female adolescents: diagnostic and clinical correlates. Psychiatry J (2013) 2013:159208. doi: 10.1155/2013/159208

52. Jacobson CM, Muehlenkamp JJ, Miller AL, Turner JB. Psychiatric impairment among adolescents engaging in different types of deliberate self-harm. J Clin Child Adolescent Psychol (2008) 37(2):363-75. doi: 10.1080/15374410801955771

53. Dillon DG, Gonenc A, Belleau E, Pizzagalli DA. Depression is associated with dimensional and categorical effects on white matter pathways. Depression Anxiety (2018) 35(5):440-7. doi: 10.1002/da.22734

54. Olson EA, Cui J, Fukunaga R, Nickerson LD, Rauch SL, Rosso IM. Disruption of white matter structural integrity and connectivity in posttraumatic stress disorder: a TBSS and tractography study. Depression Anxiety (2017) 34 (5):437-45. doi: 10.1002/da.22615

55. McCarthy-Jones S, Oestreich LKL, Lyall AE, Kikinis Z, Newell DT, Savadjiev P, et al. Childhood adversity associated with white matter alteration in the corpus callosum, corona radiata, and uncinate fasciculus of psychiatrically healthy adults. Brain Imaging Behav (2018) 12(2):449-58. doi: 10.1007/s11682-017-9703-1

56. Jenkins LM, Barba A, Campbell M, Lamar M, Shankman SA, Leow AD, et al. Shared white matter alterations across emotional disorders: a voxel-based meta-analysis of fractional anisotropy. NeuroImage Clin (2016) 12:1022-34. doi: 10.1016/j.nicl.2016.09.001

57. Mehlum L, Tørmoen AJ, Ramberg M, Haga E, Diep LM, Laberg S, et al. Dialectical behavior therapy for adolescents with repeated suicidal and selfharming behavior: a randomized trial. J Am Acad Child Adolescent Psychiatry (2014) 53(10):1082-91. doi: 10.1016/j.jaac.2014.07.003

Conflict of Interest: The authors declare that the research was conducted in the absence of any commercial or financial relationships that could be construed as a potential conflict of interest.

Copyright (c) 2020 Westlund Schreiner, Mueller, Klimes-Dougan, Begnel, Fiecas, Hill, Lim and Cullen. This is an open-access article distributed under the terms of the Creative Commons Attribution License (CC BY). The use, distribution or reproduction in other forums is permitted, provided the original author(s) and the copyright owner(s) are credited and that the original publication in this journal is cited, in accordance with accepted academic practice. No use, distribution or reproduction is permitted which does not comply with these terms. 